\title{
EDITORIAL
}

\section{A bit of antithymocyte globulins can take you a long way!}

\author{
Bone Marrow Transplantation (2012) 47, 617-618; \\ doi:10.1038/bmt.2012.6
}

The success of allogeneic hematopoietic SCT (allo-SCT) owes much to the improvements in immunosuppressive regimens that prevent GVHD. For the past 25 years, the mainstay of immunosuppression has been the calcineurin antagonists CSA and tacrolimus. However, their immunosuppressive effects can be unpredictable. The so-called ' $\mathrm{T}$ cell-depleting' antibodies such as antilymphocyte or antithymocyte globulins (ATGs) have been used for decades. The general common belief is that ATG efficacy relies on its potent capacity to deplete $\mathrm{T}$ lymphocytes of the graft. However, an ever-growing body of more recent data suggested that ATG affects not only the T lymphocytes, but also other key cells involved in the immune reaction. Indeed, the immunosuppressive activity of ATG has been thought to result primarily from the depletion of peripheral $\mathrm{T}$ lymphocytes from the circulating pool through complement-dependent lysis or activation-associated apoptosis. Other potential mechanisms of action include the modulation of surface adhesion molecules or chemokine receptor expression. In addition to their T-cell-depleting properties, ATGs can modulate the immune response by affecting or interfering with the function of different immune effectors such as B lymphocytes, regulatory $\mathrm{T}$ lymphocytes, natural killer-T lymphocytes and DCs. ${ }^{1}$

The effect of ATG on the prevention of GVHD following allo-SCT has been evaluated in several randomized controlled trials (RCTs). However, data on the efficacy of ATG use for the prevention of GVHD have been conflicting across RCTs. The most recent RCT in the field was performed by Finke et al., ${ }^{2}$ which showed that the addition of ATG-Fresenius to GVHD prophylaxis resulted in decreased incidence of both acute and chronic GVHD without an increase in relapse or non-relapse mortality, and without compromising OS. The latter prospective trial used peripheral blood-derived grafts, and required donors and recipients to be HLA compatible only at HLA-A, HLA-B, HLA-DRB1 and HLA-DQB1 (eight out of eight alleles). However, results from this prospective trial were very superimposable to the findings from the large series published by the 'Societe Francaise de Greffe de Moelle et de Therapie Cellulaire' (SFGM-TC) where the majority of donors and recipients were HLA compatible at the level of 10 out of 10 alleles and transplanted with unmanipulated BM grafts. ${ }^{3}$ In a systematic review on the efficacy of ATG for GVHD prophylaxis in 733 patients undergoing standard myeloablative allo-SCT, pooled results showed that there was a significant benefit in favor of ATG for the prevention of grade III/IV acute GVHD (risk ratio 0.51;
$95 \%$ confidence interval $0.27-0.94 ; P=0.03$ ). There was no benefit associated with ATG use for the prevention of either grade II or grade I acute GVHD. This review showed only a trend towards reduction in non-relapse mortality associated with ATG use (risk ratio $0.74 ; 95 \%$ confidence interval $0.53-1.03 ; P=0.08) .{ }^{4}$ It is important to note that studies analyzed in this systematic review differed in regard to cell source (BM vs mobilized PBSCs), donor source (matched-related vs unrelated donors vs mismatched donors), primary diseases for which allo-SCT was intended and the ablative intensity of conditioning regimens (reduced intensity vs myeloablative). In this systematic review, the beneficial findings related to the use of ATG did not appear to be impacted by a particular source of ATG or methodological quality of RCTs.

However, a recent report from the Center for International Blood and Marrow Transplant Research (CIBMTR), which examined 1676 adults undergoing reduced-intensity conditioning (RIC) allo-SCT for hematological malignancies (792 received allografts from an HLA-identical sibling, 884 from a 7 or 8 of 8 HLA-matched unrelated donor), found that disease-free survival was lower when alemtuzumab and ATG were used as part of the RIC regimen compared with T cell-replete regimens $(30 \%$, $25 \%$ and $39 \%$, respectively, $P<0.001$ ). Corresponding probabilities of overall survival were 50,38 and $46 \%$ $(P=0.008) .{ }^{5}$ On the basis of this data, the authors recommended the adoption of a cautious approach to the routine use of alemtuzumab or ATG with RIC regimens.

In this issue of the journal, Devillier et al. ${ }^{6}$ analyzed 87 patients with AML or myelodyspalstic syndrome undergoing RIC allo-SCT from an HLA-identical sibling donor. RIC consisted of Fludarabine, BU and rabbit ATG (Thymoglobuline), $2.5 \mathrm{mg} / \mathrm{kg}(n=53)$ or $5 \mathrm{mg} / \mathrm{kg}$ per day $(n=22)$. Grade $2-4$ acute GVHD incidence at day 100 was $30.2 \%$ and $8.8 \%$ in the 2.5 and $5 \mathrm{mg} / \mathrm{kg}$ groups, respectively $(P=0.038)$. Extensive chronic GVHD incidence was $60.4 \%$ and $12 \%$ in the 2.5 and $5 \mathrm{mg} / \mathrm{kg}$ groups, respectively $(P<0.001)$. The relapse incidence at 2 years was not significantly different between the two dosages. Also, OS and PFS were not different between the two dosage groups, suggesting that a relatively slight increase of the total dose of ATG from 2.5 to $5 \mathrm{mg} / \mathrm{kg}$ can make a significant difference in the setting of RIC while allowing for a good balance between acute and chronic GVHD prevention and the long-term antitumor effect.

Overall, despite its limited size, the results from Devillier et al. $^{6}$ are rather convincing. Indeed, the potential benefit of a $5 \mathrm{mg} / \mathrm{kg}$ total dose could be confirmed in other independent series from our group., ${ }^{7,8}$ This is further supported by the findings from an ongoing study by the Acute Leukemia working party of EBMT, which did not 
show a deleterious impact on the survival of ATG in a homogeneous cohort of AML patients transplanted in first $\mathrm{CR}$ and given PBSC grafts from unrelated donors (Mohty et al., unpublished data). The use of higher doses of ATG ( $>5 \mathrm{mg} / \mathrm{kg}$ total dose) and the more heterogeneous features of the study are likely to explain the negative results from the CIBMTR analysis. ${ }^{5}$

Overall, the above controversial findings clearly highlight the complexity of the clinical evaluation of ATG. Indeed, polyclonal ATG is the purified IgG fraction of sera from rabbits, horses or, more rarely, goats that are immunized with thymocytes or T-cell lines. The most widely used preparations include horse ATG (Lymphoglobulin, Genzyme, Cambridge, MA, USA) and rabbit ATG (Thymoglobulin, Genzyme). ATG Fresenius (Fresenius-BiotechGMBH, Grafelfing, Germany) includes only rabbit antibodies, and is produced against the Jurkat cell line, which resembles activated $\mathrm{T}$ cells. ATG Pharmacia (Atgam, Pharmacia Upjohn, New York, NY, USA) is derived from the horses immunized with thymocytes. Despite sharing some common properties, these different products are strictly different drugs, and have never been compared thus far in a controlled setting. Also, dose-finding studies of a given ATG brand are still lacking. Thus, one should be very cautious when deriving conclusions from the medical literature using these drugs. Obviously, the use of ATG as part of the conditioning regimen still warrants prospective investigations with respect to its impact on engraftment, immune recovery, GVHD incidence and severity, and overall outcome. The optimal dosing and timing of administration of a given ATG brand before allo-SCT are yet to be established.

Nevertheless, on the basis of the current findings by Devillier et al. ${ }^{6}$ and based on an ever-growing body of other concordant studies, and based on our own experience acquired in several hundreds of patients who received ATG as part of a RIC regimen, we can recommend that is worth to use ATG given the increasingly improved knowledge of dosages, safety and tolerance. Administration of ATG at day 0 should be avoided. Further, the use of very high doses of ATG should be avoided (for example, more than $7.5 \mathrm{mg} / \mathrm{kg}$ total dose for Thymoglobuline). A total dose of $5 \mathrm{mg} / \mathrm{kg}$ administered at $2.5 \mathrm{mg} / \mathrm{kg}$ per day at days 2 and 1 represents a good compromise that can allow for a good balance between GVHD and the graft-vs-tumor effect.

\section{Conflict of interest}

The author declares no conflict of interest.

\author{
M Mohty ${ }^{1,2,3,4}$ \\ ${ }^{1}$ Centre Hospitalier et Universitaire $(\mathrm{CHU})$ de Nantes, \\ Service d'Hématologie Clinique, Nantes, France; \\ ${ }^{2}$ Université de Nantes, Faculté de Médecine, Nantes, France; \\ ${ }^{3}$ Centre d'Investigation Clinique en Cancérologie (CI2C), \\ CHU de Nantes, Nantes, France and \\ ${ }^{4}$ INSERM CRNCA UMR 892, Nantes, France \\ E-mail:mohamad.mohty@chu-nantes.fr
}

\section{References}

1 Mohty M. Mechanisms of action of antithymocyte globulin: T-cell depletion and beyond. Leukemia 2007; 21: 1387-1394.

2 Finke J, Bethge WA, Schmoor C, Ottinger HD, Stelljes M, Zander AR et al. Standard graft-versus-host disease prophylaxis with or without anti-T-cell globulin in haematopoietic cell transplantation from matched unrelated donors: a randomised, open-label, multicentre phase 3 trial. Lancet Oncol 2009; 10: $855-864$.

3 Mohty M, Labopin M, Balere ML, Socie G, Milpied N, Tabrizi $\mathrm{R}$ et al. Antithymocyte globulins and chronic graft-vshost disease after myeloablative allogeneic stem cell transplantation from HLA-matched unrelated donors: a report from the Societe Francaise de Greffe de Moelle et de Therapie Cellulaire. Leukemia 2010; 24: 1867-1874.

4 Kumar A, Mhaskar AR, Reljic T, Mhaskar RS, KharfanDabaja MA, Anasetti C et al. Antithymocyte globulin for acutegraft-versus-host-disease prophylaxis in patients undergoing allogeneic hematopoietic cell transplantation: a systematic review. Leukemia (e-pub ahead of print 20 December 2011; doi: 10.1038/leu.2011.349).

5 Soiffer RJ, Lerademacher J, Ho V, Kan F, Artz A, Champlin $\mathrm{RE}$ et al. Impact of immune modulation with anti-T-cell antibodies on the outcome of reduced-intensity allogeneic hematopoietic stem cell transplantation for hematologic malignancies. Blood 2011; 117: 6963-6970.

6 Devillier R, Crocchiolo R, Castagna L, Fürst S, J El-Cheikh, Faucher $\mathrm{C}$ et al. The increase from 2.5 to $5 \mathrm{mg} / \mathrm{kg}$ of rabbit antithymocyte globulin dose in reduced-intensity conditioning reduces acute and chronic GVHD for patients with myeloid malignancies undergoing allo-SCT. Bone Marrow Transplant 2012; 47: 639-645.

7 Peric Z, Cahu X, Chevallier P, Brissot E, Malard F, Guillaume $\mathrm{T}$ et al. Features of Epstein-Barr Virus (EBV) reactivation after reduced intensity conditioning allogeneic hematopoietic stem cell transplantation. Leukemia 2011; 25: 932-938.

8 Malard F, Cahu X, Clavert A, Brissot E, Chevallier P, Guillaume $\mathrm{T}$ et al. Fludarabine, antithymocyte globulin, and very low-dose busulfan for reduced-intensity conditioning before allogeneic stem cell transplantation in patients with lymphoid malignancies. Biol Blood Marrow Transplant 2011; 17: $1698-1703$. 